\title{
Modeling fragment simulating projectile penetration into steel plates using finite elements and meshfree particles
}

\author{
James O'Daniel ${ }^{\mathrm{a}, *}$, Kent Danielson ${ }^{\mathrm{b}}$ and Nicholas Boone ${ }^{\mathrm{c}}$ \\ ${ }^{\mathrm{a}}$ US Army Engineer Research and Development Center, CEERD-GS-M, MS, USA \\ ${ }^{\mathrm{b}}$ US Army Engineer Research and Development Center, CEERD-GM-I, MS, USA \\ ${ }^{\mathrm{C}}$ US Army Engineer Research and Development Center, CEERD-GS-V, MS, USA
}

Received 10 April 2009

Revised 17 November 2009

\begin{abstract}
Simulating fragment penetration into steel involves complicated modeling of severe behavior of the materials through multiple phases of response. Penetration of a fragment-like projectile was simulated using finite element (FE) and meshfree particle formulations. Extreme deformation and failure of the material during the penetration event were modeled with several approaches to evaluate each as to how well it represents the actual physics of the material and structural response. A steel Fragment Simulating Projectile (FSP) - designed to simulate a fragment of metal from a weapon casing - was simulated for normal impact into a flat square plate. A range of impact velocities was used to examine levels of exit velocity ranging from relatively small to one on the same level as the impact velocity. The numerical code EPIC, used for all the simulations presented herein, contains the element and particle formulations, as well as the explicit methodology and constitutive models needed to perform these simulations. These simulations were compared against experimental data, evaluating the damage caused to the projectile and the target plates, as well as comparing the residual velocity when the projectile perforated the target.
\end{abstract}

Keywords: Penetration, finite element, meshfree/meshless methods, concrete

\section{Introduction}

Structures and vehicles have increasingly either been designed to protect their occupants from penetration of fragments or their level of protection against a variety of attacks has been assessed. These fragments typically are metal and strike the structure at a high rate of speed, attempting to penetrate and perforate the cladding of the structure and enter the interior, thereby inflicting significant damage to the structure and its occupants. This study examines the impact of a steel projectile onto flat, square, steel plates. These plates represent a basic structural component that could be used in buildings or vehicles to resist penetration and protect the occupants. Providing enhanced levels of protection for that structure and determining the geometric and material makeup used to achieve that enhancement becomes critical.

Meshfree/meshless methods, such as the Reproducing Kernel Particle Method (RKPM) [1-3] and Smooth Particle Hydrodynamics (SPH) [4-8], have been put forth as alternatives to traditional finite elements when damage and failure of a material are modeled. These particle methods have the intrinsic advantage over finite elements of not requiring element connectivity. A mesh can only break apart by either elemental removal or separation at elemental interfaces.

*Corresponding author: James O’Daniel, US Army Engineer Research and Development Center, CEERD-GS-M, 3909 Halls Ferry Road, Vicksburg, MS 39180-6199, USA. Tel.: +1 601634 3036; Fax: +1 601634 2211; E-mail: James.L.O'Daniel@ usace.army.mil. 
This has lead to various techniques that enable finite element simulations to perform calculations that include material breakup, including element deletion/erosion and the use of cohesive models that use a separation criterion and subsequent contact capabilities at element interfaces, and adaptive remeshing and/or Arbitrary Lagrangian Eulerian (ALE) methods that attempt to have the mesh better follow the breakup. Particle methods can inherently separate nodes without requiring an ad hoc technique to do so. Results from particle-based methods in EPIC [9], which used a damaged level conversion criterion that changes finite elements to particles, were benchmarked against the more traditional finite element erosion method. Both computational methods were compared to experimental data.

Many prior finite element penetration simulations have required the use of element erosion in order to allow the calculation to progress through time without severe element deformation and mesh entanglement that would dramatically lower the timestep. The existence of the mesh, or connectivity between the elements, creates difficulties when large deformations, material failure, and separation are considered. Element erosion is typically somewhat crude and very approximate; deletion of a significant portion of the continuum is typically made when some macroscopic criteria is reached, such as the effective plastic strain failure limit in steel. Another problem with finite elements is that the breakup is biased along mesh lines, i.e., element and interface directions. These are not problems with meshfree/meshless methods, as there are no elements that would need erosion or have interfaces to separate. The nature of the formulation lends itself to large deformations and failure since connectivity is not absolutely set with vertices between the nodes. The ability of the nodes to freely interact with other nodes in a meshless formulation instigated its use for penetration simulations. Of interest in this study was the damage generated by a Fragment Simulating Projectile (FSP) and the residual velocity when the projectile perforated the target. To these goals, simulations using (1) finite elements with erosion, (2) a method that converts finite elements to particles under some criteria, (3) finite elements with a section of particles in the penetration area tied to the elements, and (4) an entire target plate of particles, were performed to benchmark the methods against each other and compare against the test results.

This paper describes both the numerical simulations of the penetration scenarios and the experimental setup, compares the results from both, and summarizes the findings.

\section{Numerical models}

EPIC (Elastic-Plastic Impact Code) is an explicit, large deformation, general finite element code. EPIC has been used to solve armor penetration, earth penetration, and blast loading problems. A key feature of EPIC is its meshfree particle method, which is a modified version of SPH. The method naturally avoids mesh distortion and entanglement problems associated with finite elements and thus can model severe deformations in a Lagrangian framework. The meshless particle method, termed Generalized Particle Algorithm (GPA) was added [10] and improved upon [11] to better enable EPIC to calculate extreme material deformation and failure, and was developed to reduce many of the problems associated with the original SPH, e.g., tensile instability. The meshless-particle option includes an option to automatically convert distorted elements into particles as the solution progresses. Particle methods (including the one used in this study) are generally computationally more expensive then finite elements, but as computer speeds improve and parallel processing becomes widespread, using particle methods has become more practical and efficient. The conversion method not only reduces the computational times, but retaining elements throughout much of the deformation also reduces the severity of any tensile instability problems with the particles.

As stated, meshless methods are useful for the penetration scenarios examined here, because of their natural ability to follow very large deformations. A historically important problem of traditional finite elements in the Lagrangian formulation is that the mesh is tied to the motion of the material, which can cause elements to highly deform and distort, which in turn lead to numerical difficulties. The use of particles overcomes this problem by having the connectivity adaptable and changing as the distance between nodes increases, i.e. the weighting function decreases as the nodal range increases. The GPA differs from a traditional SPH method in that the GPA does not require the smoothing function to conform to a Dirac delta function [10]. These changes improved numerical stability for problems involving tensile stresses and improved the accuracy at boundaries and interfaces over SPH [11]. This GPA approach differs from a finite element method basically in functions used to calculate strains, strain rates, and nodal forces. The basis of both methods (finite element and meshless) in the Lagrangian formulation allows the particles 


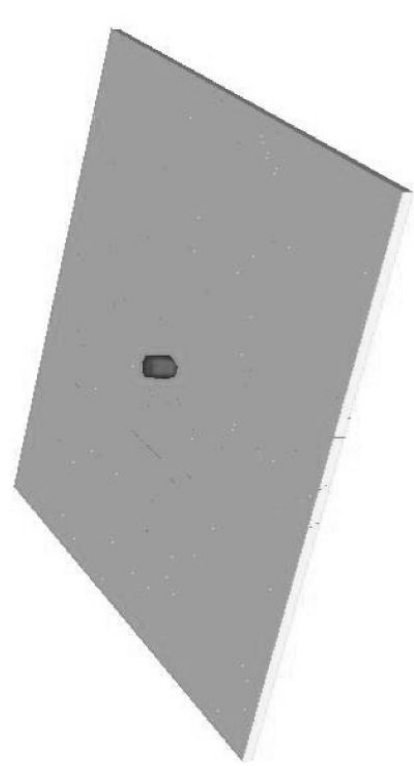

(a)

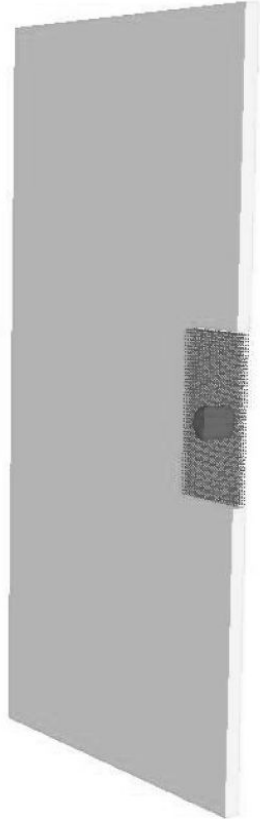

(b)

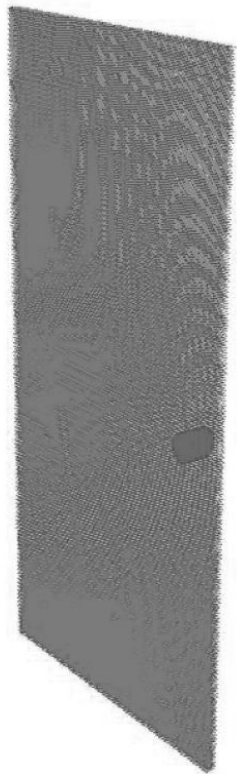

(c)

Fig. 1. (a) Finite element mesh, (b) finite element/particle mesh, and (c) particle mesh.

to be linked to elements. Since computing a mesh entirely composed of particles is much more computationally expensive than one of elements, allowing a mixed mesh of particles and elements has been developed. This includes procedures for explicitly defining different parts of the domain as particles or elements at the start of the calculation or allowing elements to convert to particles upon reaching some criteria.

Conversion of elements to particles involves the placement of a particle with the same mass and velocity as the original element at the center of gravity of that element. Once converted, contact algorithms control the interaction between particles and elements. Each particle also has a support size or influence distance over which it interacts with other particles. Once a particle leaves the influence of another particle there is no longer interaction between them $[10,11]$. This naturally allows breakup and fracture of the material through the GPA as particle motion is dictated by the physics of the problem. Erosion and conversion were performed at the same level of response within the material - at the failure strain of the steel. Material failure in the particle-only model is controlled naturally by the formulation, material properties of the target, and motion of the particles.

The target models generated for this study included a mix of elements and particles, with some models consisting entirely of elements, some entirely of particles, and some using a volume of particles in the impact area and surrounded by finite elements. Not all mesh types were simulated for every plate thickness. Figure 1 shows an example domain for a target plate for each of these model types. Tetrahedral finite elements were used in all the cases with volumetric locking avoided by a proper geometric arrangement or pressure averaging [12]. Each mesh that was initialized as finite elements contained approximately 270,000 elements with about 150, 150, and 12 in the $\mathrm{x}, \mathrm{y}$, and $\mathrm{z}$ directions, respectively. Every mesh contained two discretizations with a $50.8 \mathrm{~mm}$ by $50.8 \mathrm{~mm}$ center portion with an element size of $1.0 \mathrm{~mm}$ in the facial dimensions and $0.5 \mathrm{~mm}$ through the thickness. The remaining volume was discretized with $2.5 \mathrm{~mm}$ element size in the facial plane and the same $0.5 \mathrm{~mm}$ dimension through the thickness of the target plate. While the transition in mesh density can cause spurious wave behavior, it was deemed important to capture the precise behavior around the impact location. Mesh density was developed through preliminary simulations not described here. A finer density was used immediately surrounding the impact location. Particle densities were generated to match the finite element meshes, i.e. the number of particles in each direction approximately matched the number elements in that same direction. The physical plates/targets are described below in the experimental setup. 
Table 1

Steel material properties

\begin{tabular}{ccccc}
\hline Material & $\begin{array}{l}\text { Density, } \\
\mathrm{kg} / \mathrm{m}^{3}\end{array}$ & $\begin{array}{l}\text { Yield } \\
\text { strength, } \\
\mathrm{MPa}\end{array}$ & $\begin{array}{l}\text { Ultimate } \\
\text { strength, } \\
\mathrm{MPa}\end{array}$ & $\begin{array}{l}\text { Engineering } \\
\text { failure strain, } \\
\mathrm{mm} / \mathrm{mm}\end{array}$ \\
\hline A36 & $7,196.8$ & 248.2 & 310.3 & 0.25 \\
4340 & $7,833.4$ & $1,482.4$ & $1,578.9$ & 0.16 \\
\hline
\end{tabular}

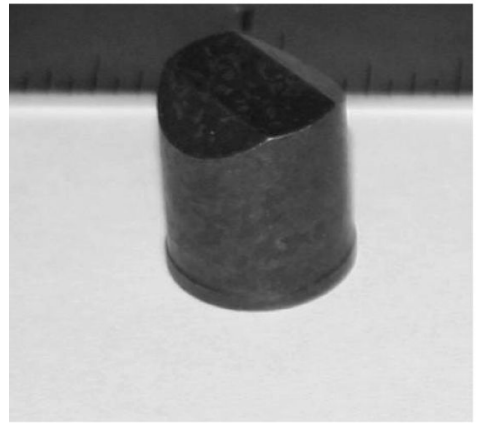

(a)

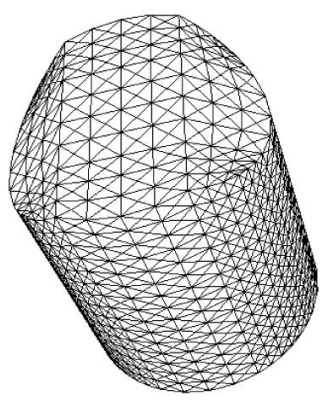

(b)

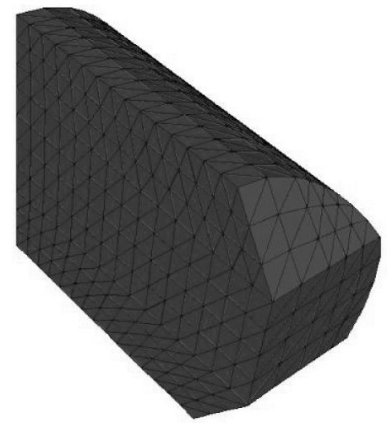

(c)

Fig. 2. Views of FSP (a) actual, (b) full, and (c) half symmetry.

Simulations initially containing only finite elements employed either erosion or element-to-particle conversion in order to examine the potential differences. When erosion was used, the finite elements were removed from the simulation upon reaching a specified strain, while for the conversion case the finite elements were changed to particles at that same specified strain level [13]. During conversion, the connectivity is removed and the mass of the element is placed into a particle at the centroid of the former element. Motion is imparted onto the particle based on the former element's momentum, and the stress state of the element moved to the particle. All of the variables are contained at the node for the particle. For all of the simulations, the projectile was initially modeled with elements, although when utilized, conversion of elements to particles was also applied to the projectile.

Nodal fixity was applied to the edges of the mesh to represent the conditions applied in the experiments. Automatic contact was used to capture the interaction between the penetrator and the target plate. This contact algorithm also ensured any interaction between failed portions of the plate would be included in the response. EPIC restricted the simulation to half symmetry when the mesh was a mix of elements and particles. Any rifling of the projectile (spin along its axis) was not incorporated into the calculations. Figure 2 shows several views of the discretization for the projectile in the full and half symmetry configurations.

Each FSP was made from 4340 steel, while the target plates were nominally A36 grade steel. Textbook [14] values were used in the simulations as coupon tests were not performed for the materials within these particular experiments. The material parameters are shown in Table 1. EPIC contains a library of material parameters and the one for 4340 steel was employed here. Steel was simulated with a Johnson-Cook metal material model, The Johnson-Cook (JC) model [15] is purely empirical and gives the following relation for the flow stress

$$
\sigma_{y}=\left[A+B\left(\varepsilon_{p}\right)^{n}\right]\left[1+C \ln \left(\dot{\varepsilon}_{p}\right)\right]\left[1-\left(T^{*}\right)^{m}\right]
$$

where $\varepsilon_{p}$ the equivalent plastic strain, $\dot{\varepsilon_{p}}$ is the plastic strain-rate, $\mathrm{T}$ is temperature, $\sigma_{y}$ is the yield stress, and $\mathrm{A}, \mathrm{B}, \mathrm{C}, \mathrm{n}, \mathrm{m}$ are material constants that describe the flow stress, strain hardening, strain rate hardening, and thermal softening. A36 steel was simulated simply by adjusting the library model parameters to reflect the different strength and ductility of that steel type. Material failure within the JC model was done through the accumulation of effective plastic strain until reaching a level defined by the engineering failure strains listed in Table 1 . This failure level was applied for both erosion of elements and conversion of elements to particles.

Simulations were performed for several plate thicknesses, varying from $6.35 \mathrm{~mm}$ to $12.7 \mathrm{~mm}$. More description of the plates is provided in the experimental description below. Of primary interest was the physical hole generated 


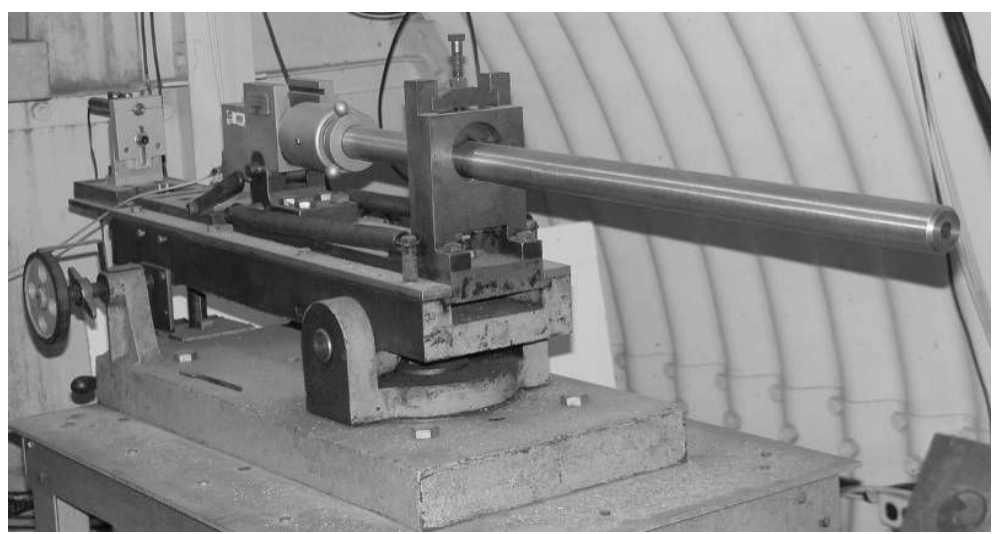

Fig. 3. Modern bond universal small-arms receiver used to fire the projectiles.

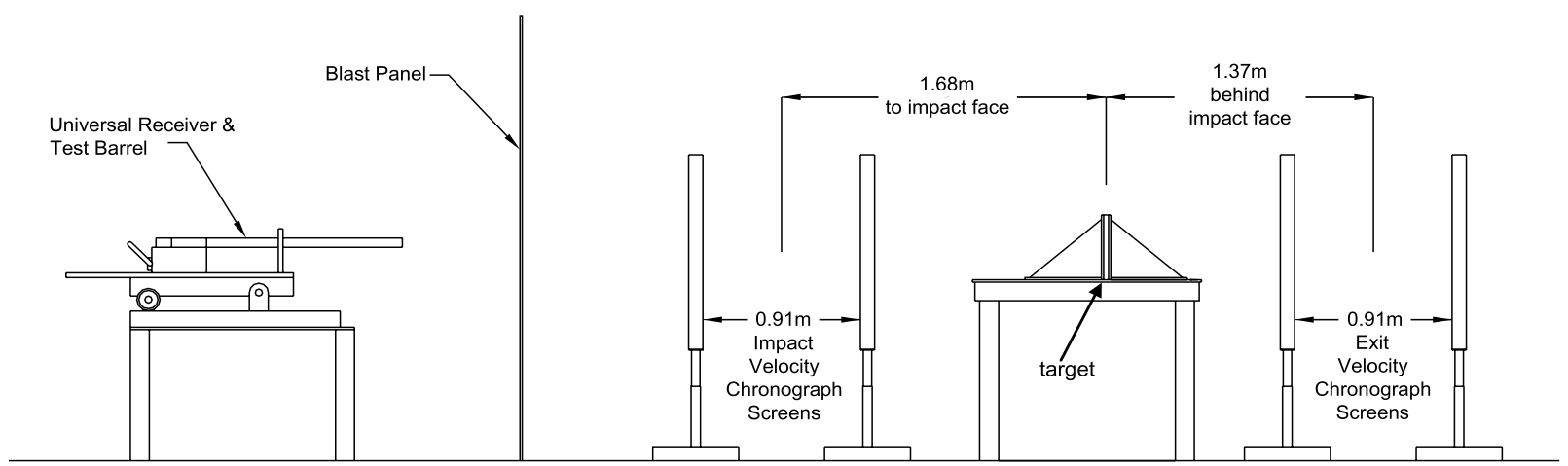

Fig. 4. Schematic of ballistic penetration test.

in the target plate by the FSP and the residual velocity of the FSP after it perforated the plate. A residual velocity of zero corresponds with the FSP not perforating the plate.

Although not explicitly included in this paper, several mesh discretizations were modeled and the one selected was deemed to have a sufficient mesh density to capture the behavior of the FSP and the plates.

\section{Experimental description}

Penetration experiments were conducted to measure the steel's resistance to ballistic penetration. Specimens tested were of uniform $304.8 \mathrm{~mm}$ by $304.8 \mathrm{~mm}$ size with approximate thicknesses of 3.81, 6.35, 9.53, and $12.70 \mathrm{~mm}$. Impact velocities were as close as the variance between tests allowed. A picture of a pristine FSP is shown above in Fig. 2. This FSP was designed to simulate a typical metal fragment. The small arms ballistic testing facility at the Engineer Research and Development Center (ERDC) consists of an underground ballistic range and an outside support building. The outside support building houses a cartridge preparation area that includes the proper equipment to handload numerous varieties of cartridges. The maximum range from muzzle to target is $8.62 \mathrm{~m}$. The range from muzzle to target in this experimental program was approximately $4.57 \mathrm{~m}$. A Modern Bond Universal small-arms receiver with a sliding return-to-zero base, shown in Fig. 3, is used to fire the projectiles. It can accommodate several barrels ranging from 0.22 caliber to 0.50 caliber.

Projectile velocity measurements were made using a pair of Oehler Research, Inc. model 35P proof chronographs, each connected to two Oehler model 55 light screens. The light screens attached to each chronograph were positioned $0.91 \mathrm{~m}$ apart to capture fragment velocities. To measure entrance and exit (residual) velocities for each experiment, 


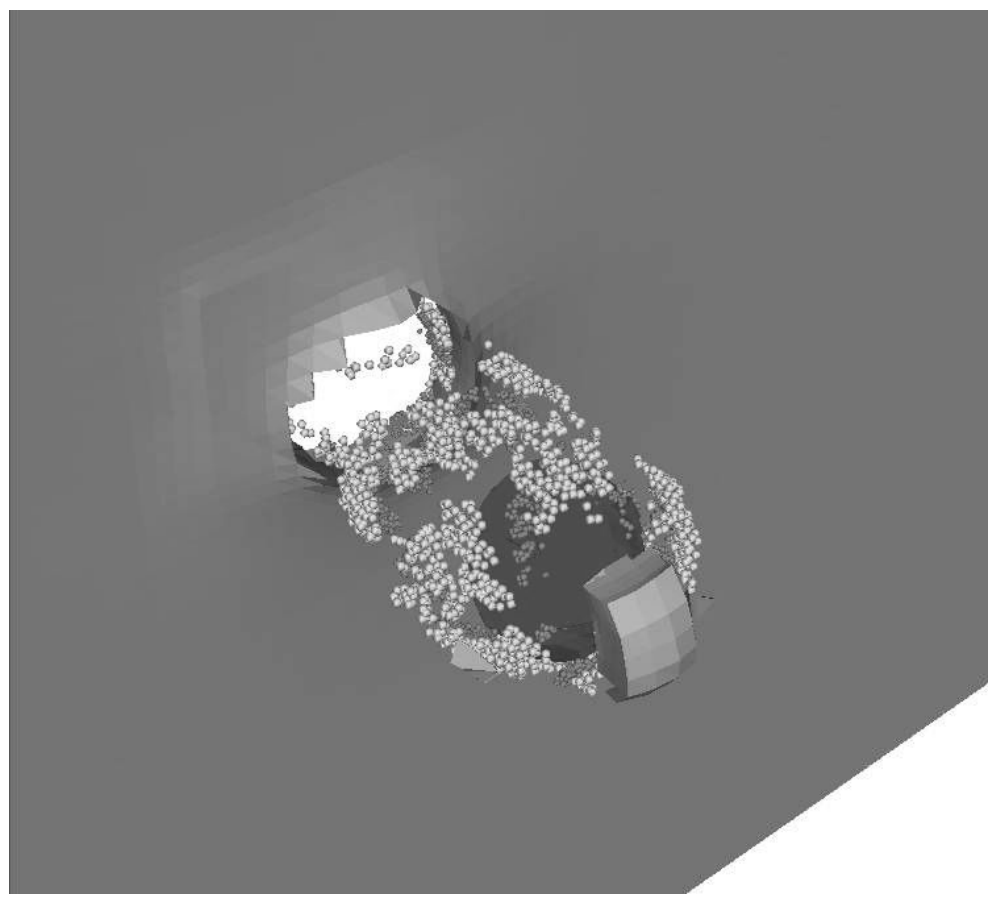

Fig. 5. FSP and particles with representative diameters.

pairs of chronograph screens (Fig. 4) were positioned so that each pair's midpoint was located approximately $1.68 \mathrm{~m}$ ahead of and $1.37 \mathrm{~m}$ behind the test specimens.

When possible, test panels were rotated 180 degrees after the first penetration test and then tested a second time near the opposite diagonal corner of the panels. The effects of the penetrator were localized and the relatively small damaged zone allowed multiple shots to be performed on each plate. Qualitative observations were also made with post-test inspection of the plate and witness panel. These observations were documented with digital photography to capture the effects of material fragments on the exit, or "safe" side of the panels.

This set of experiments included steel plates inserted into the setup described above. Each FSP was a 0.50 caliber projectile constructed of 4340 steel (with the basic properties listed in Table 1), was approximately $14.7 \mathrm{~mm}$ in length, had a diameter of about $12.7 \mathrm{~mm}$, and weighed 207 grains $(13.41 \mathrm{~g})$. The nominal impact velocity was $1,112 \mathrm{~m} / \mathrm{sec}$ with the actual impact velocity measured by the breaking of the chronograph planes by the FSP before striking the target. Those measured velocities were used as the input velocities in the simulations. Two experiments were performed for each plate thickness and the values reported below are the averages for those two tests. The only case that had a difference between the residual velocities greater than ten percent was the $12.7 \mathrm{~mm}$ thick plate, where the two exit velocities were $82.3 \mathrm{~m} / \mathrm{sec}$ and $143.0 \mathrm{~m} / \mathrm{sec}$.

\section{Results and comparisons}

Comparisons are made for all of the various plate thicknesses using the original FE mesh and conversion to particle failure method. The different methods (FE, FE-particle, and particle formulations) are only compared at the $6.35 \mathrm{~mm}$ plate thickness. Visual comparisons of the damage to the plate and the residual FSP velocity are the criteria for the comparisons. A view of the FSP perforating the target plate including the particles shown with their actual physical size representation is shown in Fig. 5. The remaining figures have the particles represented by dots at their center of gravity. Displaying the particles at actual diameter size can make the hole and debris difficult to visualize. Several views of a simulated FSP that has just perforated a steel plate are shown in Fig. 6. The FSP is colored in red, the part of the plate that has not failed is grey, and the failed parts are converted into particles, represented by 

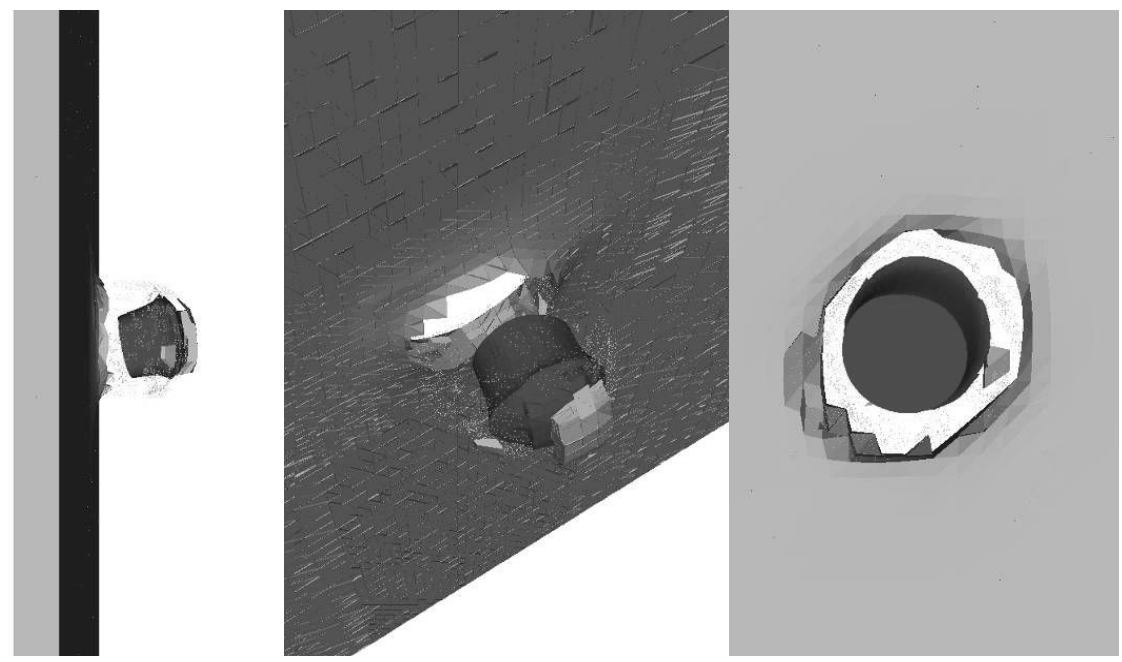

Fig. 6. Multiple views of FSP perforating a steel plate.

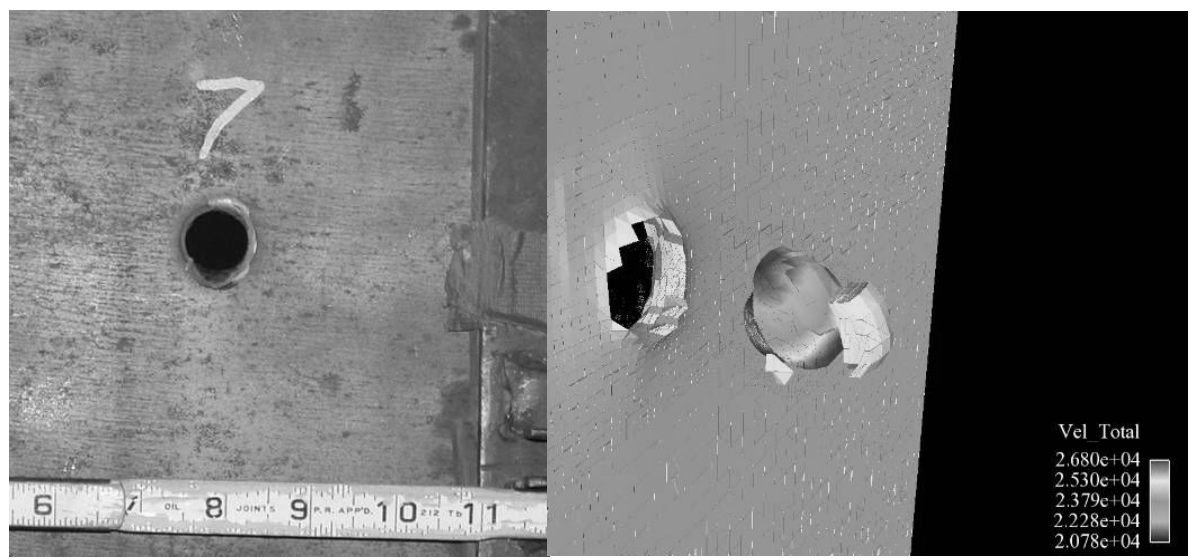

(a)

(b)

Fig. 7. Damage to the $6.35 \mathrm{~mm}$ plate (a) experiment and (b) simulation.

small dots in these figures. The FSP has turned upward slightly in Fig. 6a. This rotation was seen throughout the simulations, in both cases with and without a symmetry plane, and the reasoning behind this direction of rotation remains undetermined.

Figure 7 shows several views of the actual hole created by the FSP in the $6.35 \mathrm{~mm}$ thick plate and the numerical hole for the same test. The deformation to the FSP for that same case is shown in Fig. 8. Similar qualitative results are seen for the experiment and the simulation for both the deformation to the plate and the FSP. The FSP exhibits the same mushroom shape after exiting the target that was seen in the FSP recovered after the test.

The perforation hole size is similar in shape and size between simulations and experiments. Table 2 contains data for the hole size and the initial and residual velocities for all the tested and simulated cases. Residual velocity for the simulated FSP was estimated as the average of the velocities of its nodes, as can be seen in Fig. 7b. Figure 9 shows a plot of the residual velocities as a function of target plate thickness, containing all the simulated results and the experimental results. Error bars are plotted for the experimental results showing the minimum and maximum around the mean residual velocity for each plate.

Figure 10 shows the comparison of "damage" to the plates in each of the three simulated cases with particles and has the residual velocity bounded for the projectile. This damage is based on an accumulation of effective plastic 
Table 2

Resulting behavior of the cases

\begin{tabular}{|c|c|c|c|c|}
\hline $\begin{array}{l}\text { Plate thick- } \\
\text { ness, }(\mathrm{mm})\end{array}$ & $\begin{array}{l}\text { Initial velo- } \\
\text { city, }(\mathrm{m} / \mathrm{sec})\end{array}$ & Case & $\begin{array}{c}\text { Hole diame- } \\
\text { ter, }(\mathrm{mm})\end{array}$ & $\begin{array}{l}\text { Residual velo } \\
\text { city, }(\mathrm{m} / \mathrm{sec})\end{array}$ \\
\hline \multirow[t]{4}{*}{3.81} & 1111.91 & Experiment & 17.8 & 755.60 \\
\hline & & Numerical - FE/conversion & 18.2 & 801.12 \\
\hline & & Experiment & 19.1 & 609.90 \\
\hline & & Numerical - FE/conversion & 19.8 & 604.26 \\
\hline \multirow{3}{*}{6.35} & 1122.88 & Numerical - mixed & 21.3 & 641.86 \\
\hline & & Numerical - particles & 21.9 & 735.07 \\
\hline & & Numerical - FE/erosion & 18.2 & 678.68 \\
\hline \multirow[t]{2}{*}{9.53} & 1123.49 & Experiment & 22.9 & 346.25 \\
\hline & & Numerical - FE/conversion & 22.5 & 407.16 \\
\hline \multirow{2}{*}{12.7} & 1125.32 & Experiment & 25.4 & 112.62 \\
\hline & & Numerical - FE/conversion & 22.8 & 304.04 \\
\hline
\end{tabular}

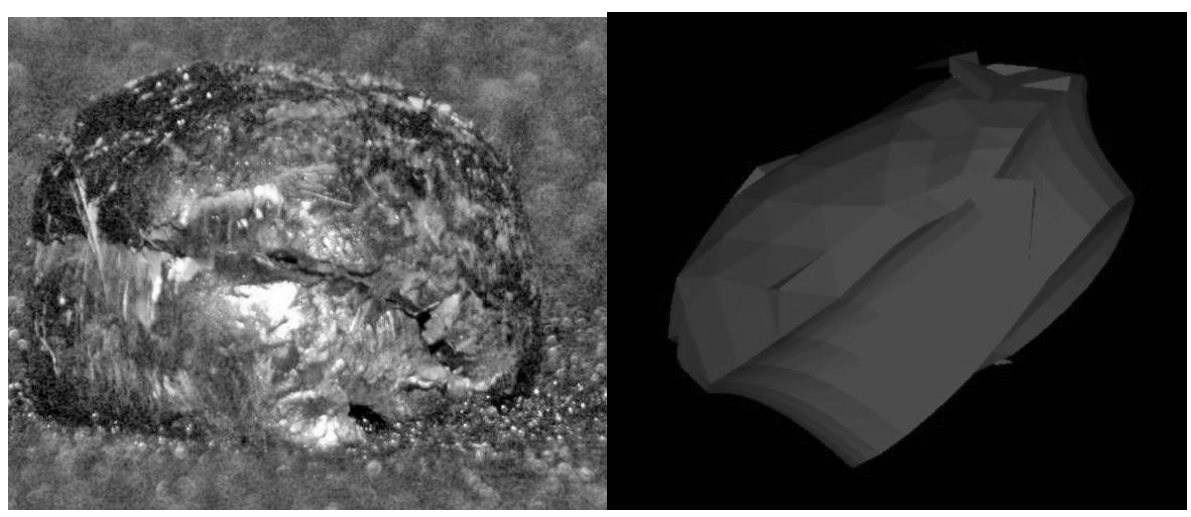

(a)

(b)

Fig. 8. Comparison of deformed FSP (a) experiment, and (b) simulation.

strain. The scale shown for velocity (V_scale in the plots) applies to the penetrator only, while the scale for damage only applies to the target plate. Damage is set on a scale from zero to 1.0 (which is approximately the failure strain). The velocity is higher when a mesh consisting entirely of particles was used, while the residual velocity from the other two cases was relatively close. Damage to the plate was consistent throughout the simulations, exhibiting very little damage away from the hole generated in the target.

Using only particles for the $6.35 \mathrm{~mm}$ plate produced larger holes than were seen in the cases starting with finite elements. Hole sizes compared well when conversion to particles was used as the failure method for plate thicknesses up to $12.7 \mathrm{~mm}$. For the $12.7-\mathrm{mm}$ thick-plate simulation both the residual velocity and the hole size did not compare as well as it did for the thinner panels. The FSP perforated through the plate and numerically had almost twice the residual velocity as the highest exit velocity in the experiments. As the plate thickness is increased and the residual velocities become smaller (i.e. the FSP gets closer to not going through the plate) the response is not being as accurately captured using the same techniques and failure criteria that produced good comparisons for the thinner plates. As the major response seems to be a punching shear or plugging in the thinner plates, the hole sizes were similar, and the response did not tear the plate or cause visible macrocracking to propagate away from the penetration hole. The discrepancy found between the $12.7 \mathrm{~mm}$ thick-plate experiments and simulations may be caused by not modeling the transition of failure modes from initial piercing, characterized by radial hydrodynamic flow, to a plugging phenomenon. It is likely that the piercing mode occurs during the initial penetration until some critical depth is reached where the remaining target thickness is capable of failing by plugging. Indications of both failure modes are present in the post-test photos and are shown in Figs 11a and 11b. Figure 11a presents the impact face and illustrates the tapered hole shape indicative of radial flow. Figure $11 \mathrm{~b}$ shows the back face which looks nearly identical to the thin plate shown in Fig. 7 and therefore is also believed to have failed by plugging or shear. 


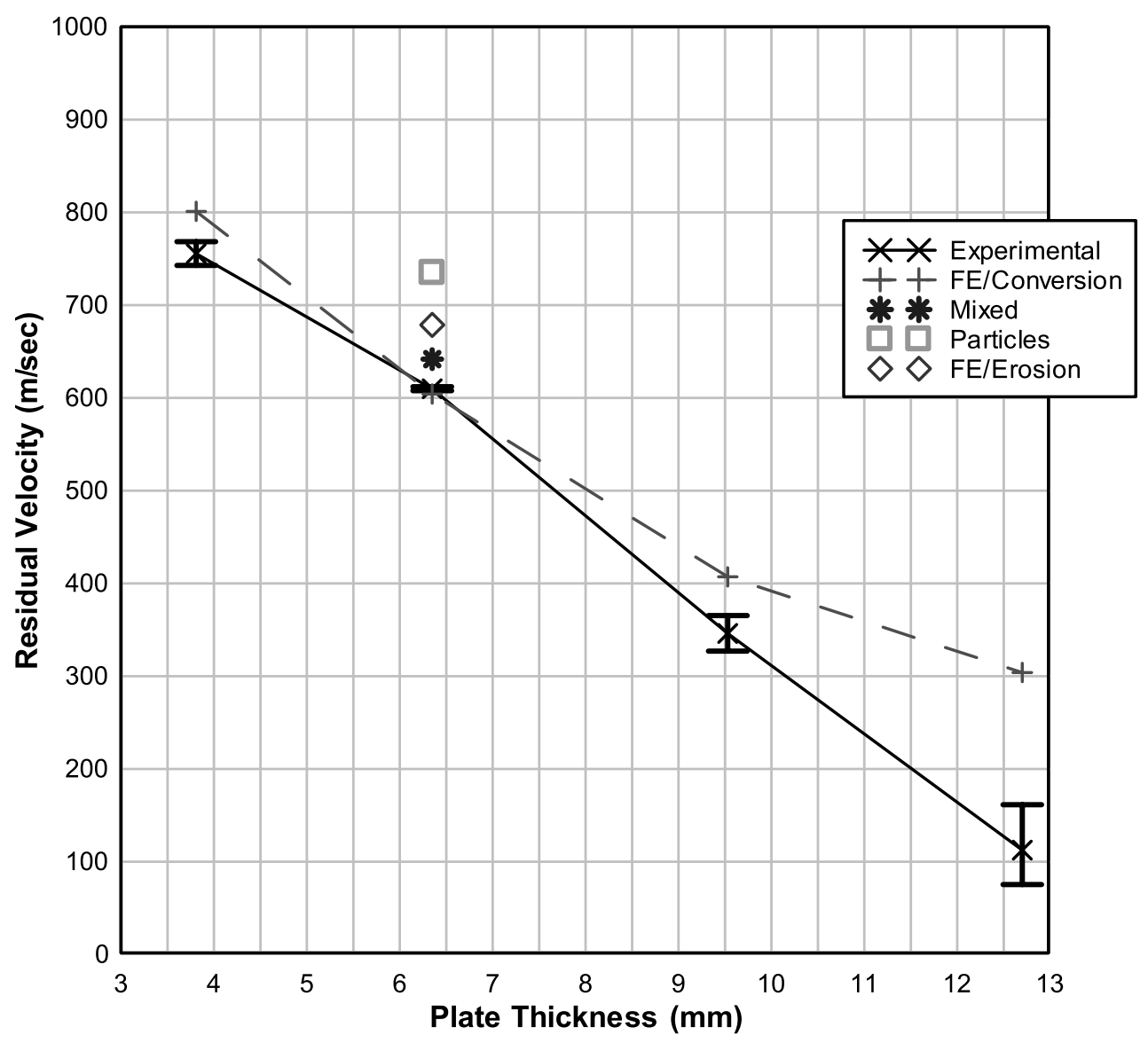

Fig. 9. Plot of residual velocities.

Better results were achieved with the simulations for the thinner plates, with the residual velocities only differing by $6.0 \%$ for the $3.81 \mathrm{~mm}$ plate and $1.0 \%$ for the $6.35 \mathrm{~mm}$ plate (compared between the experiments and the FEconversion case). This comparison was not as good for the $9.53 \mathrm{~mm}$ plate $(\sim 17.5 \%$ difference) and the simulations predicted a significantly larger exit velocity for the $12.7 \mathrm{~mm}$ plate. Note that, as mentioned above, the greatest discrepancy in the repeated tests was also in the $12.7 \mathrm{~mm}$ thick plate. Note also that the over-predicted residual velocities increased with increased plate thickness. This might indicate an error in the plate material properties.

A comparison was made in the total energy within the plate for each of the four simulated cases with a plate thickness of $6.35 \mathrm{~mm}$; FE with erosion, FE with conversion, partial particle, and a complete plate of particles. This comparison is shown in Fig. 12. As it should, the plate energy is lower when erosion is used, removing energy from the simulation with each element removal. Interestingly, when the entire plate consists of particles, the energy is lower by $8-10 \%$ than when conversion is used or when part of the target plate is particles. These two cases exhibit similar levels of energy within the plate.

\section{Summary}

Experiments and simulations were performed to evaluate the ability of several numerical methods to model the penetration of an FSP into and through steel plates of several thicknesses. The FE code EPIC allowed these cases to be modeled using only finite elements (with erosion and conversion failure techniques), using a combination of finite elements and particles, and using a mesh containing only particles. Comparisons were made between the different 


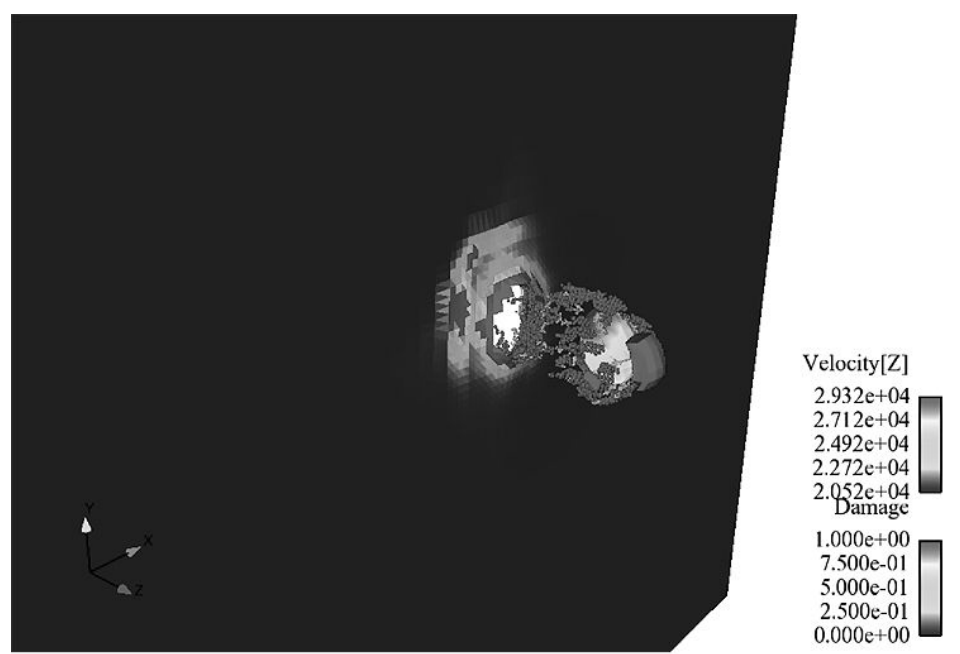

(a)

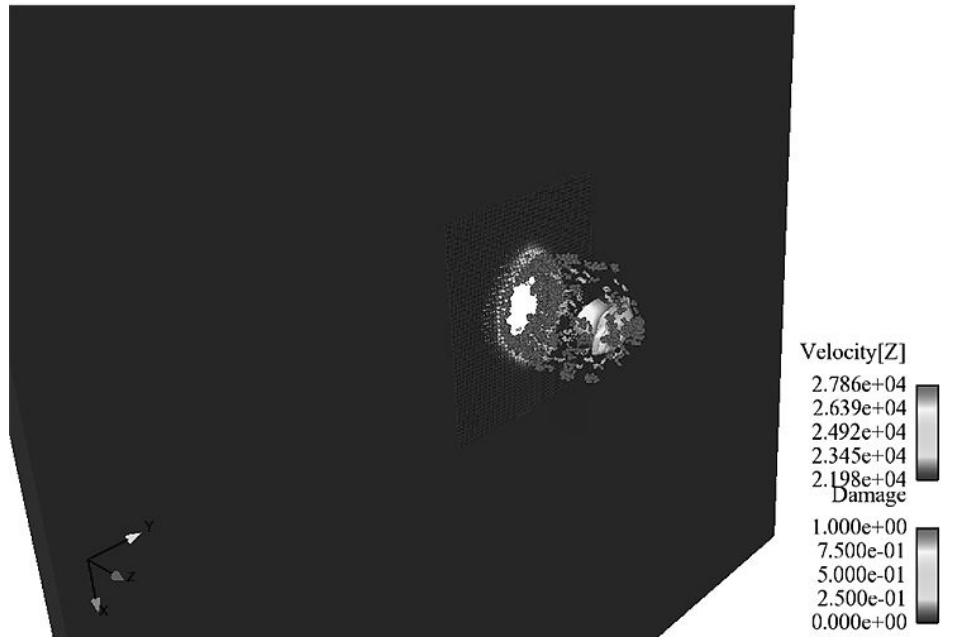

(b)

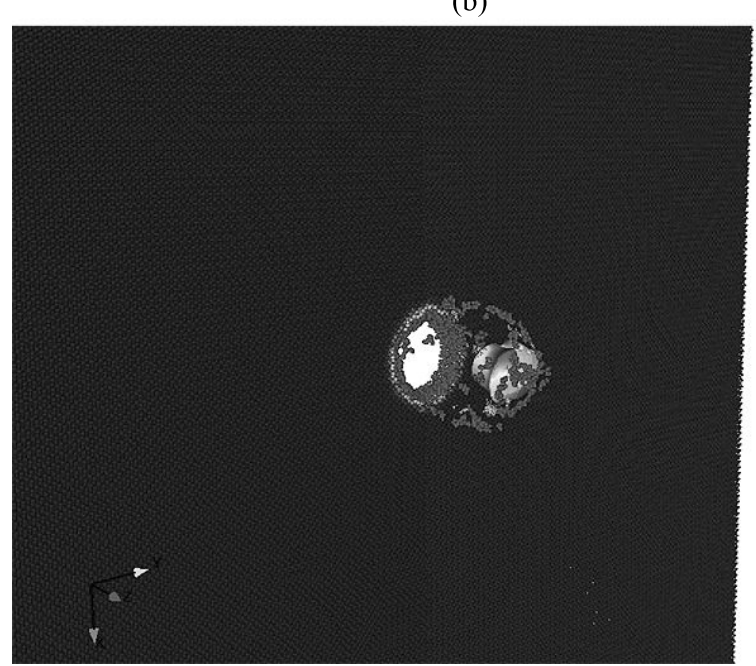

Damage
$1.000 \mathrm{e}+00$
$7.500 \mathrm{e}-01$
$5.000 \mathrm{e}-01$
$2.500 \mathrm{e}-01$
$0.000 \mathrm{e}+00$
Velocity[Z]
$3.090 \mathrm{e}+04$
$2.983 \mathrm{e}+04$
$2.876 \mathrm{e}+04$
$2.769 \mathrm{e}+04$
$2.661 \mathrm{e}+04$

(c)

Fig. 10. Projectile penetration and panel damage for (a) conversion case, (b) combined particle/element mesh, and (c) total particle mesh. 


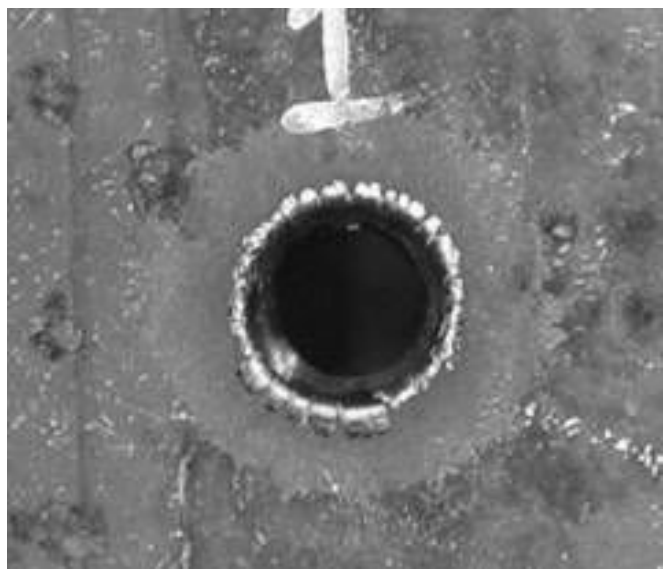

(a)

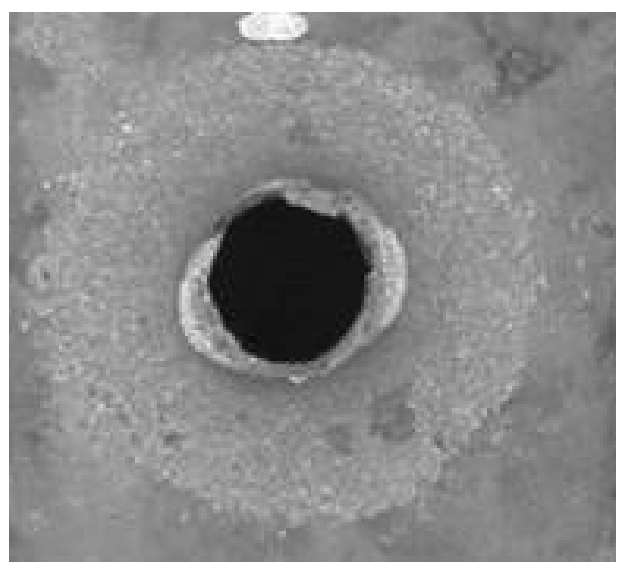

(b)

Fig. 11. $12.7 \mathrm{~mm}$ plate failure modes (a) piercing impact, and (b) plugging exit.

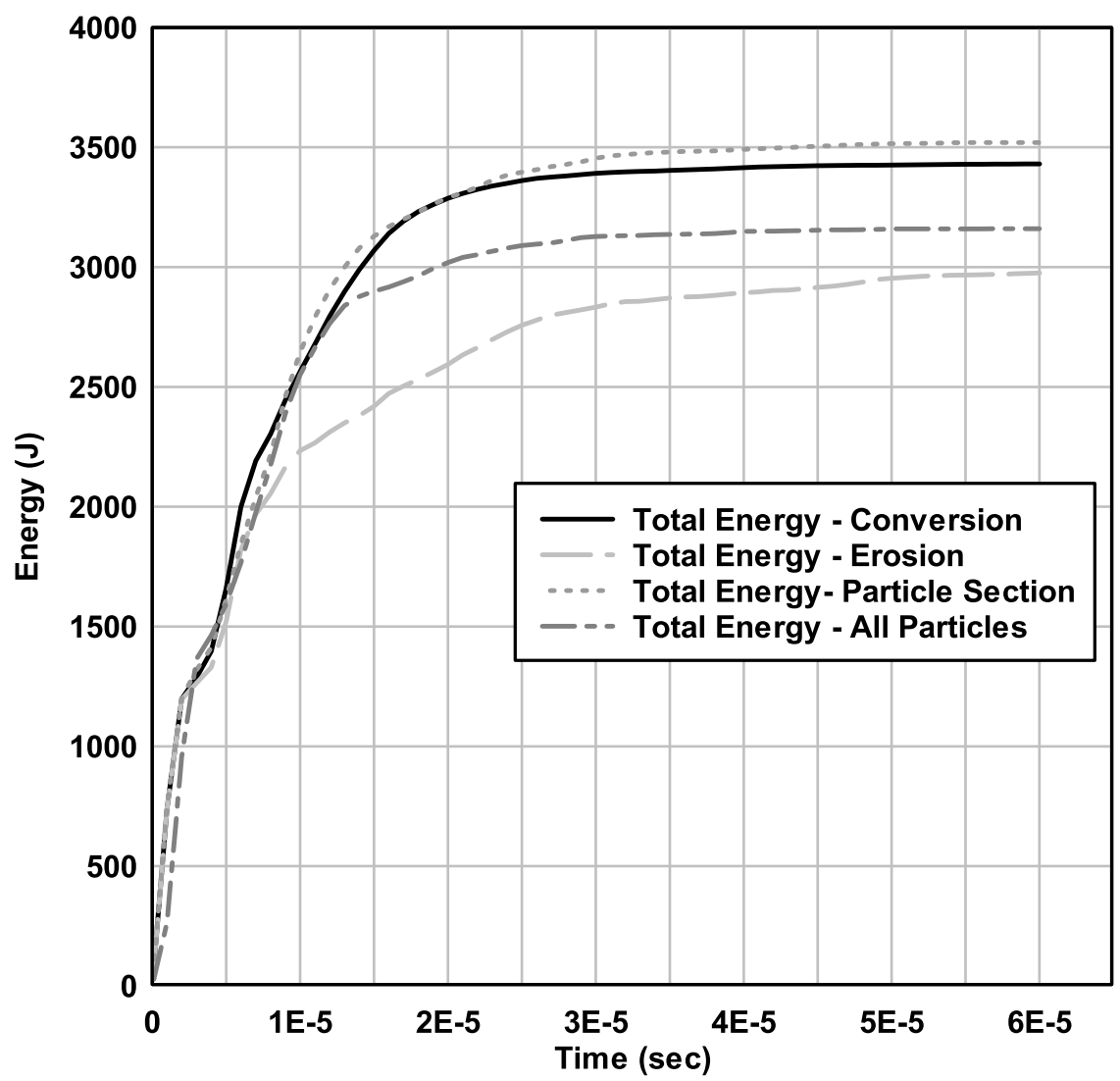

Fig. 12. Total energy of the $6.35 \mathrm{~mm}$ thick target plate. 
formulations and the experimental results for gross damage to the target plate and for residual velocity of the FSP. A comparison of the energy within the plate was also made for only the numerical cases.

Best results were achieved when the finite elements were converted to particles using a plastic strain criterion. Good correlation was obtained for the thinner plates between numerical and experimental cases for residual velocity, damage to the plate, and FSP response. It appears that there are noticeable tensile instability inaccuracies in cases that started with only a partial particle mesh. Further investigation is needed to determine the discrepancy between simulation and experiment as the thickness of the target plate increased, which might also require obtaining better target material properties. Although there is still room for improvement for this very challenging application, the finite element-conversion to particle method does seem to be better than either the erosion finite element or particles without conversion methods.

\section{Acknowledgements}

Simulations done in support of this project were performed on the systems of the Engineer Research and Development Center Major Shared Resource Center. Permission to publish was granted by Director, Geotechnical and Structures Laboratory.

\section{References}

[1] W.K. Liu, W. Hao, Y. Chen, S. Jun and J. Gosz, Multiresolution reproducing particle methods, Computational Mechanics 20(4) (1997), 295-309.

[2] W.K. Liu, T. Belytschko and J.T. Oden, Meshless Methods, Computer Methods in Applied Mechanics and Engineering, Vol. 139, Issue 1, 1996.

[3] J.S. Chen and W.K. Liu, Meshfree Particle Methods, Computational Mechanics 25, num 2, 2000.

[4] L.B. Lucy, A numerical approach to the testing of fusion process, The Astronomical Journal 88 (1977), 1013-1024.

[5] R.A. Gingold and J.J. Monaghan, Smoothed particle hydrodynamics: theory and application to non-spherical stars, Monthly Notices Royal Astronomy Society 181(1977), 375-389.

[6] W. Benz, Smooth particle hydrodynamics: a review, Harvard-Smithsonian Center for Astrophysics, (Preprint 2884$), 1989$.

[7] J.J. Monaghan, Smoothed particle hydrodynamics, Annual Review Astronomics and Astrophysics 30 (1992), $543-574$.

[8] L.D. Libersky and A.G. Petschek, Smooth particle hydrodynamics with strength of materials, Advances in the Free Lagrange Method, Lecture Notes in Physics, Springer: Berlin, Vol. 395 (1990), 248-257.

[9] G.R. Johnson, S.R. Beissel, C.A. Gerlach, R.A. Stryk, T.J. Holmquist, A.A. Johnson, S.E. Ray and J.J. Arata, User Instructions for the 2006 Version of the EPIC Code, Final Report, Contract DAAD19-03-D-0001, U.S. Army Research Laboratory, 2006.

[10] G.R Johnson, S.R. Beissel and R.A. Stryk, A generalized particle algorithm for high velocity impact computations, Comput Mech 25 (2000), 245-256.

[11] G.R Johnson, S.R. Beissel and R.A. Stryk, An improved generalized particle algorithm that includes boundaries and interfaces, Int J Numer Meth Eng 53 (2002), 875-904.

[12] G.R Johnson, D.D. Colby and D.J. Vavrick, Three-dimensional computer code for dynamic response of solids to intense impulsive loads, Int J Numer Meth Eng 14, 1979.

[13] G.R Johnson and R.A. Stryk, Conversion of 3D distorted elements into meshless particles during dynamic deformation, Int J Numer Meth Eng 28(9) (October 2003), 947-966.

[14] Defense Special Weapons Agency (DSWA), Design and Analysis of Hardened Structures to Conventional Weapons Effects, Technical Manual, TM 5-855-01, AFPAM 32-1147(1), NAVFAC P-1080, DAHSCWEEMAN-97, 1998.

[15] G.R Johnson and W.H. Cook, A constitutive model and data for metals subjected to large strains, high strain rates and high temperatures. In: Proc. $7^{\text {th }}$ International Symposium on Ballistics, 1983, pp. 541-547. 

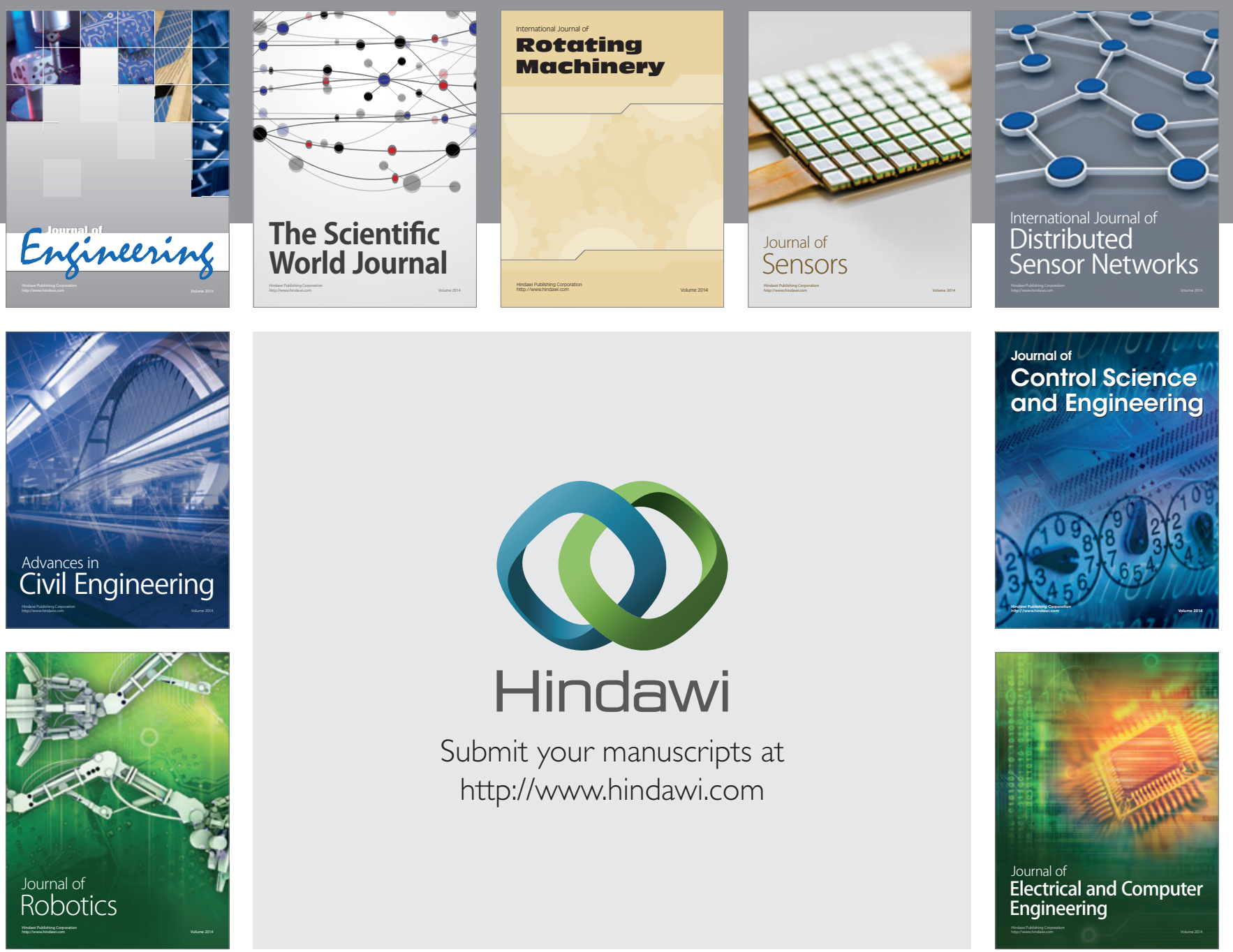

Submit your manuscripts at

http://www.hindawi.com
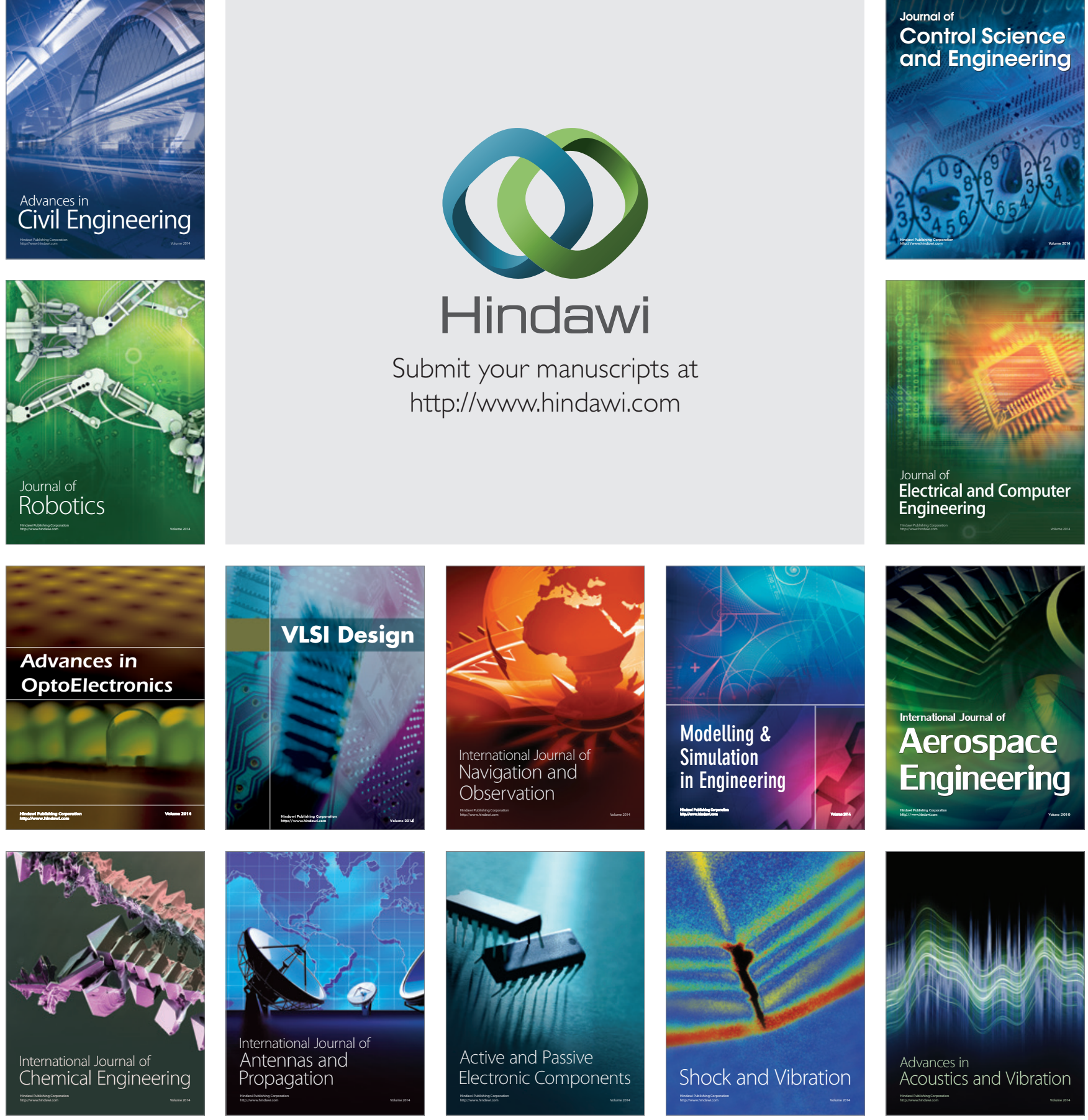